\title{
Challenges of Interaction in Multicultural Class of Future Mining Engineers within Computer Supported Collaborative Learning
}

\author{
Svetlana Kolomiets ${ }^{1,{ }^{*}}$, Irina Saveleva ${ }^{1}$, and Elena Medvedeva ${ }^{2}$ \\ ${ }^{1}$ Kemerovo State University, Institute of Philology, Foreign Languages and Media Communication, \\ Department of Foreign Languages, 650000, Krasnaya Street, 6, Kemerovo, Russia \\ ${ }^{2}$ Kemerovo State Medical University, Foreign Students Office, 650056, 22A Voroshilova st., \\ Kemerovo, Russia
}

\begin{abstract}
The inclusion of computer mediation and distance education has influenced the training of future mining engineers in Kuzbass universities. This study investigates the issues of students' interaction in multicultural groups within Computer Supported Collaborative Learning. CSCL served as a conceptual framework to guide the presented study. Forty-eight Russian and Indian students participated in the experiment. To assess the peer interaction in multicultural groups four questionnaires were designed. The authors used comparative analysis to analyze the interviews of the participants. To process the data, the Pearson's correlation analysis was performed. In the result of comparative analysis of the participants' interviews some conclusions on students' interaction in multicultural class were done. Foreign students demonstrate positive attitude towards collaborative learning activities while one in two Russian students displays reluctance and frustration in relation to cross-cultural collaborative learning environment. The results suggest that the main challenges for implementation of CSCL in higher education institutions of Kuzbass are insufficient cross-cultural skills of students and even teachers and the lowquality digital infrastructure of universities.
\end{abstract}

\section{Introduction}

In the last decade, modern society has been rapidly changing almost every day. The changes taking place in the industrial enterprises of Kuzbass are inseparably connected with fast developing technologies, globalization and the coronavirus pandemic. Additionally, technological convergence - the result of accelerating scientific and technological progress in the 21 st century - has a transformative effect on the economies of developed countries, forming a new type of sectorial genesis [1]. Due to these changes, the managers and engineers of Kuzbass industrial enterprises frequently work on international projects collaborating with their colleagues through the Internet. The demands of the modern information age compel higher education graduates and those people who already have working place to be equipped

*Corresponding author: kolomsvetlana@yandex.ru 
with the 21 st century skills. This set of skills will allow professionals to achieve great results in their career and to make a significant contribution to society or industry [2].

That's why the employers seek in their future employees such skills as:

- critical thinking;

- communication;

- creativity;

- collaboration.

In order to participate in and succeed in today's rapidly changing workplace, future engineers need to acquire appropriate technical skills alongside with developed interpersonal communication skills. Moreover, nowadays lots of technical universities graduates can take internship or practice in another country where they meet the realities of a multi-cultural community with different ways of knowledge transfer, different educational regulations, social and corporate ethics standards and responsibilities [3].

Therefore, the primary task of universities is to develop effective learning environment to help students meet the demands of a new century. Equipping future engineers with crosscultural skills is equally important as they study and after graduation when they will work in the international environment.

This requires the implementing of new teaching approaches, adjusting the process of study to the modern situation. Although, there are many different ways in which any classroom can be changed to meet the needs of modern education, technology plays a leading role in shaping present-date classes. Modern technologies provide educators with various tools for promoting the 21 st century skills to prepare students for their future careers in the industrial enterprises of Kuzbass. At the disposal of university teachers there are different web-sources and mobile applications that have become an inherent part of educational process. Modern technologies have changed the 21 st century learning environment and made it more flexible. In its turn, flexible learning environment allows teachers and student to make choices about the learning activities and settings - online as well as offline. The pandemic of Covid-19 forced most of educational institutions to give a crucial part to distance learning while each educational institution chose some peculiar set of teaching methods and learning patterns to organize an online environment. The set includes national and international platforms with MOOCs and webinar services, social networks and messengers, and e-mail newsletters.

To prepare students for the 21 st century skills the majority of university teachers choose the approach of computer supported collaborative learning. It is increasingly becoming an instructional approach of choice in both the traditional face-to-face and online education settings. Computer Supported Collaborative Learning (CSCL) refers to learning situations mediated by technologies where small groups of 3 to 5 students are exposed to interaction in order to solve a complex unstructured problem or are required to design a project [4]. Computer-Supported Collaborative Learning is the learning process when students work together to achieve joint learning goals by communicating amongst themselves via the Internet, and where student-to-student interaction plays a primary role in the learning process.

For current research, it is necessary to outline the difference between cooperation and collaboration as these notions are frequently confused and describe the specific features of computer supported collaborative learning.

Collaboration is a philosophy of interaction and personal lifestyle where individuals are responsible for their actions, including learning and respect the abilities and contributions of their peers. In all situations where people come together in groups, it suggests a way of dealing with people that respects and highlights individual group members' abilities and contributions [5].

Meanwhile cooperative learning refers to learning that takes place in situations where students are required to work in small groups, usually under the direct guidance of the 
instructor, who may set specific tasks and objectives for each session. Most models of cooperative learning employ intentional grouping stipulated by the instructor, or random assignment. Computer-Supported Collaborative Learning never uses assigned group roles, but the majority of cooperative learning approaches do this. Cooperative learning emphasizes teamwork, while collaborative learning emphasizes individual performance in teams. According to the new constructivist theory, cooperative learning emphasizes the traditional backward "teacher as the main body", while collaborative learning emphasizes "students as the main body" $[6,7]$.

In this way, within cooperative learning approach classroom is still mostly teachercentered and students are deprived of learning choices and cannot realize their creative and collaborative skills. On the contrast, CSCL helps to create a learning environment where students themselves under the supervision of a teacher construct their knowledge.

CSCL approach is primarily distinguished by necessary implementation of modern technologies in an educational process. Actually CSCL was designed in response to the requirements for modern learning environment to be high-tech and accessible online. CSCL blends learning and technology built on collaborative work but it does not mean the interaction and collaboration of learners only in an online communication medium. The computer support may involve, for instance, a computer simulation of a scientific model or a shared interactive representation. In this case, the collaboration focuses on the construction and exploration of the simulation or representation. Alternatively, a group of students might use a computer to browse through information on the Internet and to discuss, debate, gather and present what they found collaboratively [8].

The media should be based on student's needs and learning objectives. Today there is a great choice of sources that can support students' interaction and collaboration during their work on projects. Such online platforms as Google Classroom, Padlet, Socrative, Blackboard, Twilddla, Scribblar, Nearpod, various educational blogs and social media provide effective, easy to use, and a quite often free of charge for connecting students and teachers interested in pursuing collaborative learning. Within CSCL approach educators frequently employ them as they provide:

- online chat;

- space to hold online discussions;

- sharing of images, email, and documents;

- a feedback tool to provide an assessment of students' work.

It can be specifically noted that students prefer to share learning artifacts frequently and voluntarily using for this purpose social networks technologies. It is associated with prosocial motives and collectivist values and is slightly less pronounces in competitive situations [9].

The second specific characteristic of CSCL is the collaboration of students. Within the given approach, students' learning takes place largely through interactions among the students. Students learn by expressing their questions, solving problems from different perspectives and resolving communicative problems, which may arise during their interaction. Thus, students generate new knowledge via transforming individual ideas into collective experience. In collaborative communities new knowledge is formed within three intellectual phases:

- idea-generating;

- idea organization and

- intellectual convergence [10].

Yet, alongside with the perspectives offered by the use of CSCL there are some challenges concerning the organization of it. The main problem is determined by the cultural diversity of the individual learners. The researchers mention that the following aspects are significantly affected by the cultural background of the learners:

- motivation; 
- attitude towards learning and e-learning;

- learning styles;

- computer usage in education;

- learning behavior and strategies;

- academic achievements;

- communication;

- participation;

- knowledge transfer, sharing and collaborative learning [11].

Among the communication problems posed before the culturally diverse learners there have been distinguished:

- inability to understand specific cultural references in online discussions;

- lack of non-linguistic cues;

- difficulties expressing disagreement;

- communicative constraints resulting in less substantive postings;

- mismatched communication patterns [12].

To sum it up, the successful implementation of CSCL in training of future engineers depend on the following factors:

- technologies and resources;

- learning environment;

- participants'communicative skills;

- the experience of teachers and students in CSCL.

\section{Methods}

Today the educational landscape in Kuzbass is greatly affected by the challenges of the 21 st century. Multicultural classes and online education are a common feature of all higher education institutions in Kemerovo region. Students from different countries choose universities of Kuzbass as their abroad study destination. The presence of culturally diverse students in different (online and offline) classroom settings compel university teachers to implement new approaches and technologies into an educational process.

CSCL is a relatively new strategy in teaching multicultural class of future engineers in Kuzbass universities. Therefore, there are no sufficient and reliable data concerning how the practices of CSCL fit in multicultural learning environment of universities in Kuzbass. It is extremely important to understand in what way teachers with varying pedagogical experience and students with varying skills and experiences are able to function in computer supported collaborative learning environment.

The given study was aimed to assess the perception of culturally diverse students towards interaction during collaborative learning activities supported by modern technologies. The related goal was to examine the digital infrastructure of universities to provide computer supported collaborative activities for their students and the students' choices of Internet resources to execute collaboration with groupmates. Presumably, the findings of the study can suggest the educators what aspects should be taken into consideration to design effective collaborative learning activities for culturally diverse students.

To achieve the goals of the study the experiment was designed and done. The focus of the research was put on the following aspects of students' interaction within CSCL learning:

- the impact of settings (online/offline) on the students' progress;

- collaboration and interaction with groupmates during online and regular classes;

- teacher's support during online and regular classes;

- time-management during online and regular classes.

The experiment was divided into three stages described in Table 1. 
Table 1. The stages of the experiment.

\begin{tabular}{|c|c|c|}
\hline Stage & Substance & Goals \\
\hline $\begin{array}{c}\text { First stage } \\
\text { "Assignment } \\
\text { task" }\end{array}$ & $\begin{array}{c}\text { Students were given a task } \\
\text { to make a joint project } \\
\text { with the support }\end{array}$ & $\begin{array}{c}\text { The aim was to provide } \\
\text { students with clear } \\
\text { instructions for the } \\
\text { assignment and some } \\
\text { guidelines on their } \\
\text { collaborative work via } \\
\text { modern technologies }\end{array}$ \\
\hline $\begin{array}{c}\text { Second stage } \\
\text { "Project work" }\end{array}$ & $\begin{array}{c}\text { Students worked on and } \\
\text { demonstrated their } \\
\text { projects, assessed the } \\
\text { other projects }\end{array}$ & $\begin{array}{c}\text { The goal was to observe } \\
\text { students' activities and } \\
\text { interaction during the } \\
\text { collaborative work on the } \\
\text { project }\end{array}$ \\
\hline $\begin{array}{c}\text { Third stage } \\
\text { "Opinion poll" }\end{array}$ & $\begin{array}{c}\text { Students were asked to fill } \\
\text { out four questionnaires }\end{array}$ & $\begin{array}{c}\text { The purpose was to analyze } \\
\text { the students' attitudes } \\
\text { towards the collaborative } \\
\text { assed their readiness for } \\
\text { interaction in a multicultural } \\
\text { environment }\end{array}$ \\
\hline
\end{tabular}

\subsection{Participants}

Participants were first and second year students studying in industrial universities of Kuzbass. There were 36 Russian and 12 international students. All the students successfully demonstrated their knowledge of English and were able to use it as a lingua franca for communication. The students who studied in regular classes and those who studied online were divided into small groups of three students from different cultural backgrounds. Thus, the groups had one student from one culture and two others from another one.

\subsection{Assignment and CSCL procedure}

The assignment used in this study was to be done within the module of Business English. Business English Module is integrated in the English Language Course that is a part of regular study program in Russian universities. The aim of this module is to develop students' communication skills in the sphere of business. All the students that participated in the study were well equipped with necessary knowledge and skills to cope with given assignment. The students were assigned to develop a joint project.

The task for the students was to design a new product to be launched by one of Kuzbass enterprise and its video presentation. The new product had to respond to the needs of people living and working in Kemerovo region.

To conduct the task and do the project, the students were asked to exchange ideas in small groups both during online and regular classes. Outside the class, they were offered to interact within small groups in online settings. They could choose any resources for online interaction such as Padlet and Blogspot.com or any social media and mobile applications. Students were 
encouraged to use any available Internet sources, YouTube videos and relevant posters and pictures during their collaborative work on the project. Presentations were discussed and assessed via the interactive board Padlet. During the collaborative work of students, the teacher provided only a few comments on students' projects and did not contribute to the students' interaction or comment on the students' knowledge productions. The teacher performed a role of facilitator and co-learner.

The CSCL procedure involved two stages. In the first stage the students were randomly divided into groups of three-four students from different cultural groups. In such a manner, both the students who studied online and those who studied in regular classes were divided into small groups.

In the second stage all the students were given the assignment and the guidelines that were posted in the online learning platform Moodle. The students had 24 hours access to this resource and could get teacher's assistance via this online platform.

Based on theoretical framework and goals of the study field, some questionnaires were designed. Upon completion of the project, the students answered the questions of these short questionnaires. The questionnaires were aimed to assess and analyze the interaction between students from different cultures in online and regular classes within CSCL approach. Participants were asked to fill out four questionnaires. Each of the questionnaires contained eight questions. The survey was conducted by 12 foreign and 36 Russian students (1st and 2nd year students engaged in 12 groups of 4 people: six teams of the first year and six of the second year of study).

The questions of the first questionnaire were aimed at establishing a general attitude (General attitude / GA Variable 1) to collaborative projects and assessing learners' academic achievement as a result of participation in the projects. The purpose of the second questionnaire was to identify the relationship between traditional, contact forms of interaction in the classroom and online interaction (Contact learning / CL Variable 2). The third questionnaire assessed the students' use of visual aids in the course of the project (Visual aids / VA Variable 3). The fourth questionnaire was aimed at the perception of the time limit applied in collaborative learning (Timing / T Variable 4). To process the data, the Pearson's correlation analysis performed in the Statistics 12 software was used.

\section{Results}

The analysis of the questionnaires data has shown some convergences and divergences in the assessment of interaction and collaboration demonstrated by the participants from Russian and Indian cultures.

Russian students (Table 2) had an average score of $45 \%$ for the first variable (perception of online collaboration), while for the foreign students it was $84.5 \%$ (Table 3 ). The average values for the second variable for Russian (Table 2) and foreign students (Table 3) are approximately equal (37 and $36.7 \%$ respectively). The average indicator for the third variable reveals a significant gap (63 and 92\% respectively), and the fourth indicator (53 and $30 \%$ ) shows significant differences in the assessment of time component of collaborative learning.

The establishment of correlational relationships (according to the Cheddock's scale) showed a strong correlation between the general attitude to interaction in the composition of projects and contact forms of learning (0.789) among the Russian students. According to the data collected in the group of foreign participants, strong correlations with retroactive effect are observed between the general attitude to collaborative learning and contact forms of interaction (-0.991), as well as between the first test indicator and the time limits set for project participants (-0.994). A positive correlation in the analysis of the responses of foreign 
students is observed between the contact interaction with the teacher and the time constraints of the project work (0.976).

Table 2. Russian students' perception of collaborative learning: mean, standard deviation and correlation coefficients $(\mathrm{P}<0.05, \mathrm{~N}=36)$.

\begin{tabular}{|c|c|c|c|c|c|}
\hline Variable & Mean & Std. Dev. & $\mathbf{1}$ & $\mathbf{2}$ & $\mathbf{3}$ \\
\hline 1 - GA & 45.332 & 23.501 & - & - & - \\
\hline $2-\mathrm{CL}$ & 37.312 & 18.796 & 0.789 & - & - \\
\hline 3 - VA & 63.193 & 10.519 & 0.369 & 0.494 & - \\
\hline $4-\mathrm{T}$ & 53.392 & 14.929 & 0.320 & 0.391 & 0.421 \\
\hline
\end{tabular}

Table 3. Indian students' perception of collaborative learning: mean, standard deviation and correlation coefficients $(\mathrm{p}<0.05, \mathrm{~N}=12)$

\begin{tabular}{|c|c|c|c|c|c|}
\hline Variable & Mean & Std. Dev. & $\mathbf{1}$ & $\mathbf{2}$ & $\mathbf{3}$ \\
\hline 1 - GA & 84.583 & 23.179 & - & - & - \\
\hline 2 - CL & 36.690 & 20.043 & -0.991 & - & - \\
\hline 3 - VA & 91.666 & 15.012 & 0.696 & -0.637 & - \\
\hline $4-\mathrm{T}$ & 30.208 & 22.168 & -0.994 & 0.976 & -0.771 \\
\hline
\end{tabular}

Analyses of similarity and variance in participants' feedback were conducted to compare and analyze participants' perceptions on interaction during computer supported collaborative learning activities.

The observation showed the students preferred to use mobile devices with visualization applications and some of interactive capabilities to support their collaborative activities. Participants chose Moodle platform only to get teacher's support and some instructions. During their collaborative learning some students created virtual network community of students in Instagram, Facebook and VKontakte.

\section{Discussion}

The interpretation of the average values for each of the variables allows us to establish the following.

The positive attitude of foreign students towards collaborative projects is clearly expressed, while Russian students are divided into approximately two equal groups in their opinions.

Both Russian and foreign students are equally interested in the participation and the facilitative role of the instructor, and also note the lack of contact forms of interaction and interpersonal communication during the implementation of online projects. The auxiliary role of visual tools used in the implementation of joint projects in social networks is more highly appreciated by foreign students, which indicates the need to use a large number of illustrative supports (images, animations, fonts of different colour, and size, etc.) when teaching this category of students. One of the findings was the aversive attitude of foreign students to 
deadlines and the introduction of time restrictions in the implementation of these projects. It is more difficult for them to fit the prescribed time limits and to be able to submit the completed work on time. The pace of work of foreign students is slower, so the inclusion of them in teams with Russian students made it more difficult to perform the work quickly. This perception of deadlines in online learning is directly proportional to the contact interaction with the teacher. The inverse relationship observed between the general attitude to collaborations and self-assessment of one's academic performance in the context of collaborative learning, which is particularly pronounced in foreign students, may indicate a preference for interaction in real mode.

The participants also note the difficulties they have experienced during their collaborative work in a multicultural triad. The students who had to interact and collaborate only in online settings stress such drawbacks as dependence on the Internet connection, some technical problems, and the lack of experience in creating joint projects. Some students felt frustrated in intercultural collaboration that led to unequal participation in the project work. Students studying online also mention the lack of non-verbal interaction.

Both Russian and Indian students who combined face-to-face and online interaction identify such drawbacks of their collaborative work as sociocultural tension during the interaction with peers, lack of group participation in the collaborative work and divergent modes of communicating, interacting and working.

All students come to the same conclusion that online collaboration requires substantial time to achieve the understanding between the group members. They mentioned the experienced time management problems among the other challenges and barriers of interaction during their collaborative activities.

The study has helped find out the benefits, preferences, and challenges of students from diverse cultural backgrounds as they participate in online and offline collaborative learning activities.

The analysis of the data identified the following challenges of integrating CSCL in training of multicultural groups:

- unreadiness of students to work without clear instructions of a teacher:

- different learning styles of students;

- the lack of common ground between culturally diverse students;

- challenges of dealing with cultural differences;

- the lack of necessary technology equipment in the universities;

On the other hand, the implementation of CSCL in multicultural class can lead to such academic and social benefits as the following ones:

Academic benefits:

- CSCL promotes critical thinking and collaborative skills;

- CSCL develops students' technology and information literacy;

- CSCL involves students actively in the learning process;

- CSCL creates cross-cultural communication among students;

- CSCL enhances students' motivation in specific curriculum.

Social benefits:

- CSCL develops a social support for learners from different cultural backgrounds;

- CSCL builds cultural diversity understanding among students and teachers;

- CSCL establishes a positive atmosphere for modelling and practicing collaboration;

- CSCL develops learning communities.

Students master their collaborative and communication skills and develop their computer and information literacy. Working in a small multicultural team, they are also exposed to cross-cultural communication and gain experience of communication in team-based learning environment. 


\section{Conclusion}

The inclusion of CSCL in training of future engineers can better prepare the students for their future careers in a virtual and multicultural work environment. Although, CSCL leads to students' self-development, improving learning quality, sharing knowledge and assisting students' in the process of building their knowledge, implementation of CSCL demands technology relevant facilities and teaching methods for promoting student collaboration.

In general, students have a positive perception towards the collaborative learning in a multicultural environment and the usage of technologies for learning activities. Meanwhile the results of the research indicate that some cultural characteristics of participants as an attitude to the learning and teamwork, pace of work and perception of time prevent collaboration and hinder effective learning interaction.

\section{Acknowledgement}

The reported study was funded by RFBR according to the research project № 19-013-00805

\section{References}

1. S. Zhironkin, M. Cehlar V. Zhironkin, E3S Web Conf. 134, 03013 (2019)

2. I. Saveleva, O. Greenwald, S. Kolomiets, E. Medvedeva, E3S Web Conf. 21, 04024 (2017)

3. J. Kretschmann, I. Pevneva, S. Ivanova, E. Sant'eva, M. Bakanov, E3S Web Conf. 174, 04035 (2020)

4. D. W. Johnson, R. T. Johnson, ER 38(5), 365-379 (2009)

5. M. Laal, S. M. Ghodsi, Procedia - Social and Behavioral Sciences, 31, 486-490 (2012)

6. N. Davidson, C. H. Major, Journal on Excellence in College Teaching, 25(3\&4), 7-55 (2014)

7. J. Wu, IJET, 13(07), 10.3991 (2018)

8. G. Stahl, AI\&SOCIETY, 14(1), 71-97 (2000)

9. E. Bouton, S. Bar Tal, C.S.C. Asterhan, The Internet and Higher Education, 49, 100787 (2021)

10. A. Kumi-Yeboah, J. Dogbey, G. Yuan, OLJ, 21(4), 10.24059 (2017)

11. A.A. Economidies, IJME, 2(4), 243-267 (2008)

12. V. Popov, H. Biemans, D. Brinkman, A. Kuznetsov, M. Mulder, The Internet and Higher Education, 19, 36-48 (2013) 